\title{
Role of Oxygen Defects on Photoelectrochemical Activity of ZnO Nanorods Grown on Transparent Conducting Oxide Thin Film
}

\author{
Hasmat Khan, Srikrishna Samanta, Malobi Seth, and Sunirmal Jana* \\ Specialty Glass Technology Division, CSIR-Central Glass and Ceramic Research Institute, \\ 196 Raja S. C. Mullick Road, Jadavpur, Kolkata 700 032, India \\ Email: sjana@cgcri.res.in, janasunirmal@hotmail.com (S. J.)
}

\begin{abstract}
ZnO}$ nanorods (NRs) were grown onto ZnO seed layer deposited FTO glass substrate via hydrothermal technique at $\sim 95^{\circ} \mathrm{C}$. The as-deposited samples were further heat-treated at $\sim 400{ }^{\circ} \mathrm{C}$ in a furnace under air atmosphere. With varying time, the kinetic study of the growth of $\mathrm{ZnO}$ towards NRs formation was also performed. To enhance oxygen defects such as oxygen vacancy in the NRs, the thermally cured samples were further heat-treated in $5 \% \mathrm{H}_{2}$ gas atmosphere at $\sim 350{ }^{\circ} \mathrm{C}$. Morphological and microstructural analyses, estimation of oxygen vacancy in the samples were carried out by field emission scanning and transmission electron microscopies and X-ray photoelectron spectroscopy, respectively. Moreover, the optical properties of the specimen were also characterized by photoluminescence spectral study. A co-relation was also made between the reduced gas treatment time and the photoelectrochemical (PEC) activity of the samples. The ZnO NRs synthesized by optimizing the time for the growth of NRs and the reduced gas treatment time exhibited significant improvement in PEC activity. This work could make an avenue for enhancing the PEC activity of other hierarchically structured metal oxide semiconductors.
\end{abstract}

Keywords hydrothermal growth technique, $\mathrm{ZnO}$ nanorods, morphology and microstructure, oxygen defects, photoelectrochemical activity

\section{Introduction}

Nowadays, the development of advanced energy materials is very much needed for generation of clean energy from sustainable energy sources. ${ }^{[1,2]}$ To fulfil the energy demand, the production of clean energy (hydrogen fuel) from water splitting reaction with the help of solar light is one of the ways. ${ }^{[1]}$ In photoelectrochemical (PEC) reaction, semiconductor materials are the key component for solar water splitting via generation of photoexcited electron-hole pairs. ${ }^{[2]}$ To improve the device performance, the semiconductor materials with improved optoelectronic property have a great importance. ${ }^{[2,3]}$ Among various metal oxide semiconductors (MOSs), $\mathrm{ZnO}$ is a promising MOS for PEC application due to its cost effectiveness, non-toxicity and earth abundant in nature. ${ }^{[4]}$ The deposition of nanostructured $\mathrm{ZnO}$ film on different substrates is possible by various techniques, among which hydrothermal growth technique is an effective one. ${ }^{[5]}$ Moreover, one-dimensional (1D) nanostructures such as nanowires and nanorods with direct electron transport capability can also provide large surface area that is beneficial for improving PEC activity of ZnO..$^{[5,6]}$

It is well-known that the n-type character of $\mathrm{ZnO}$ semiconductor is governed by the surface defects such as oxygen vacancies and interstitial zinc that can act as deep donor and shallow donor, respectively. ${ }^{[6]}$ Enthalpy of the formation of these defects is very low. ${ }^{[7,8]}$ The defect can accept holes from valence band and becomes single ionised oxygen vacancy or doubly ionized oxygen vacancy. ${ }^{[6,8]}$ Such single/doubly ionised oxygen vacancies are responsible for lattice distortion, which facilitates efficient charge separation during the PEC process. ${ }^{[6,9]}$ In this regard, various approaches such as noble metal doping and the formation of heterostructures with other MOSs towards generation and separation of photoexcited charges had already been reported by several authors. ${ }^{[1,10]}$ Our group $^{[11]}$ also reported singly negatively charged oxygen vacancy in solution based $\mathrm{Al}$ doped $\mathrm{ZnO}$ thin film as an efficient photocatalyst for decomposition of organic dye under visible light irradiation. Yang et al. ${ }^{[10]}$ worked on $\mathrm{Ag}$ nanoparticles coated sandwiched $\mathrm{CdS} / \mathrm{Ag} / \mathrm{ZnO}$ heterostructures photoanode for improved light absorption and efficient charge separation during the PEC water splitting reaction. However, the enhancement of performance of PEC water splitting reaction by inducing oxygen vacancies in the MOS can be considered an efficient, straightforward and cost effective route.

In the present work, one-dimensional $\mathrm{ZnO}$ nanorods (NRs) were grown on the seeded FTO glass substrate via hydrothermal technique. The kinetic study on the growth of $\mathrm{ZnO} N R \mathrm{~s}$ was also performed at different refluxing time. Deposited $\mathrm{ZnO}$ films were further heat-treated at $400{ }^{\circ} \mathrm{C}$ under air atmosphere in a tube furnace. To increase oxygen vacancy in the ZnO NRs, the heat-treated samples in air were further cured in $\mathrm{H}_{2}$ gas atmosphere at $350{ }^{\circ} \mathrm{C}$. Morphology and microstructure as well as the oxygen vacancy present in the samples were analysed by the field emission scanning electron microscopy, transmission electron microscopy, photoluminescence spectroscopy and X-ray photoelectron microscopy. A co-relation was also made between the reduced gas treatment time and the PEC activity of the samples. The ZnO NRs synthesized by optimizing the time for the growth of NRs as well as the reduced gas treatment time exhibited significant improvement in the PEC activity of the sample.

\section{Experimental}

\section{Precursor materials}

Zinc nitrate hexahydrate, $\left[\left(\mathrm{Zn}\left(\mathrm{NO}_{3}\right)_{2} \cdot 6 \mathrm{H}_{2} \mathrm{O}, \mathrm{ZN}\right.\right.$, E. Merck, assay $\geq 98 \%$, zinc acetate dihydrate $\left[\mathrm{Zn}\left(\mathrm{CH}_{3} \mathrm{COO}\right)_{2} \cdot 2 \mathrm{H}_{2} \mathrm{O}, \mathrm{ZA}\right.$ Sigma-Aldrich, $\geq 98 \%$ ], hexamethylenetetramine (HMTA, Merck, 
assay $\geq 99 \%$ ), methanol (Merck, assay $\geq 99 \%$ ), potassium hydroxide pellets AR (S. D. Fine-Chem Pvt., Ltd.) were used as received without their further purifications for preparation of the precursor solutions.

\section{Preparation of $\mathrm{ZnO}$ nanorods}

\section{ZnO seed layer on FTO glass substrate}

Initially, $20 \mathrm{~mL}$ ZA solution $(0.2 \mathrm{mM})$ in methanol was prepared under vigorous stirring at $60{ }^{\circ} \mathrm{C}$. Then, $12 \mathrm{~mL} \mathrm{KOH}$ solution $(0.35 \mathrm{mM})$ in methanol was added dropwise into the solution. The solution was continuously stirred for $2 \mathrm{~h}$ at $60^{\circ} \mathrm{C}$. After that, a dip-coating technique (dip coater: Chemat Technology Inc., Dip Master 200, USA) was performed to deposit $\mathrm{ZnO}$ seed layer onto the cleaned fluorine doped tin oxide (FTO; Product code- TISXZ 001; Techinstro, India; dimension: length $25 \mathrm{~mm}$, width $25 \mathrm{~mm}$, thickness $2.2 \mathrm{~mm}$ and resistivity $\sim 7$ ohms/sq) and pure silica glass (Quartz glass plate, code CUSQ223; Ants Ceramics Pvt., Ltd., India; dimension: length $75 \mathrm{~mm}$, width $25 \mathrm{~mm}$ and thickness $\sim 1 \mathrm{~mm}$ ) substrates using the precursor solutions. The films were further cured at $400{ }^{\circ} \mathrm{C}$ for $1 \mathrm{~h}$ in a tube furnace under air atmosphere.

\section{Deposition of $\mathrm{ZnO}$ nanorods onto the seed layer}

$\mathrm{ZnO}$ nanorod arrays on pure silica FTO glass substrate were deposited by the modified method of a reported work. ${ }^{[12]}$ In brief, ZN $(0.595 \mathrm{~g})$ and hexamethylenetetramine $(0.35 \mathrm{~g})$ were added in $50 \mathrm{~mL}$ distilled water and the solution was stirred for 2 $\mathrm{h}$ at ambient condition. Then, $30 \mathrm{~mL}$ of the solution was transferred into a $50 \mathrm{~mL}$ tarson tube and the $\mathrm{ZnO}$ seed layer coated cured films were placed inside the tube for hydrothermal reaction. The solution was refluxed at $95{ }^{\circ} \mathrm{C}$ with variation of refluxing time of $0.5,1$ and $2 \mathrm{~h}$. Subsequently, the deposited films were carefully rinsed with distilled water and cured at $400{ }^{\circ} \mathrm{C}$ for better film adherence and crystallinity. The deposited film was designated as ZNR. To create the oxygen vacancies in the $\mathrm{ZnO}$ nanorods, the ZNR samples were further heat-treated at $350^{\circ} \mathrm{C}$ in reduce gas atmosphere $\left(5 \% \mathrm{H}_{2}\right)$ and the films were termed as $\mathrm{ZNRH}$. An overall film fabrication process is schematically displayed in Scheme 1.

Scheme 1 Schematic presentation for the growth of $\mathrm{ZnO}$ towards formation of nanorods on $\mathrm{ZnO}$ seed layer coated FTO glass substrate

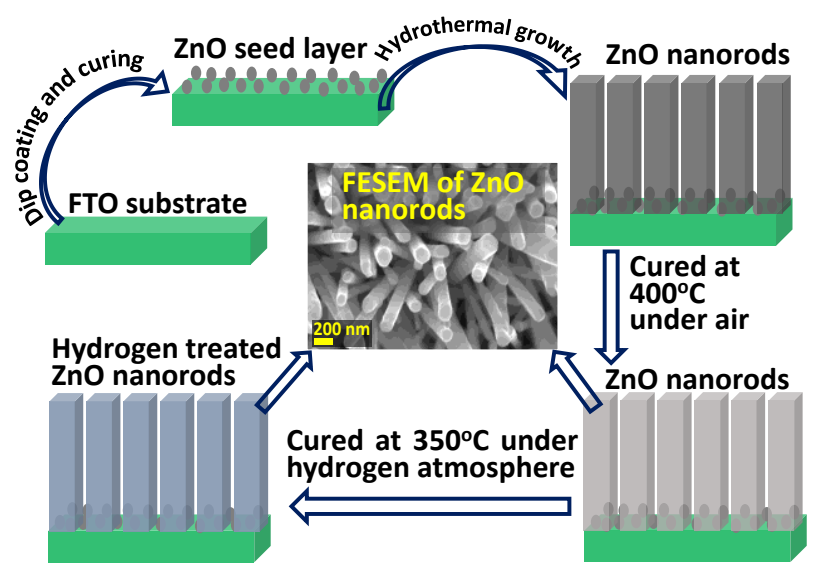

\section{Characterizations}

\section{Materials properties}

To understand the crystal phase/crystallinity of ZNR films, the grazing incidence X-ray diffraction (GIXRD) study was performed by using Rigaku Smart Lab, Japan make diffraction unit employing $\mathrm{Cu}$ Ka radiation (1.5406 $\AA$ ) operating at $9 \mathrm{~kW}$ in the diffraction angle range $(2 \theta)$ of $10^{\circ}$ to $80^{\circ}$. The GIXRD measurement was performed with a slit width of $5 \mathrm{~mm}$. Surface features of the films were analysed with the help of field emission scanning electron microscope (FESEM and FESEM-EDS, ZEISS, SUPRA ${ }^{\mathrm{TM}}$ 35VP). Transmission electron microscopic (TEM) study was also performed by Tecnai $\mathrm{G}^{2}$ 30ST (FEI company, Netherlands) at an accelerating voltage of $300 \mathrm{kV}$. Carbon coated 300 mesh $\mathrm{Cu}$ grids were used for the preparation of samples. The X-ray photoelectron spectra (XPS) of the representative samples, ZNR and $Z N R H$ were measured to know the oxidation state of constituent elements as well as the relative contents of oxygen deficiencies with the help of $\mathrm{PHI}$ Versaprobe II Scanning XPS microprobe surface analysis system using Al-Ka X-rays ( $\mathrm{h} v, 1486.6 \mathrm{eV} ; \Delta E, 0.7 \mathrm{eV}$ at room temperature). Pure Ag sample was used to calibrate the energy scale of the spectrometer. The position of $\mathrm{C} 1 \mathrm{~s}$ peak was taken as standard (binding energy, $284.5 \mathrm{eV}$ ). A Perkin-Elmer (LS55) spectrofluorimeter was used to measure the photoluminescence (PL) spectra of ZNR and ZNRH samples at room temperature in the wavelength range of $350-650 \mathrm{~nm}$ at a fixed excitation wavelength $340 \mathrm{~nm}$. For this purpose, pure silica glass substrate was used for the deposition on ZnO NRs under identical condition as done on FTO substrate. Light harvesting efficiency of the samples was measured with the help of UV-Vis-NIR spectrophotometer (Shimadzu, UV-3600) with an attachment of an integrating sphere (ISR-3100, UV-PC-3100).

\section{Photoelectrochemical activity}

Metrohm, Autolab AUt 85930 instrument with standard three-electrode cell was used to measure the photoelectrochemical (PEC) activity of ZNR and ZNRH samples deposited onto FTO coated glass substrate under dark as well as visible light exposure. For this purpose, a $300 \mathrm{~W}$ Xenon lamp with a water filter of $1 \mathrm{M} \mathrm{NaNO}_{2}$ solution was used as the visible light $\left(\geq 400 \mathrm{~nm}\right.$ ) source ${ }^{[13]}$ For measurement of the PEC activity, a Pt wire and an $\mathrm{Ag} / \mathrm{AgCl} / 3 \mathrm{M} \mathrm{KCl}$ electrode were taken as the counter electrode and reference electrode, respectively. The fabricated ZNR and ZNRH samples were used as a working electrode. An aqueous solution of $0.1 \mathrm{M} \mathrm{Na}_{2} \mathrm{SO}_{4}$ was used as an electrolyte. The chronoamperometric study (photocurrent density vs. time, $l-t$ curve) of the samples was carried out at $0.62 \mathrm{~V}$ vs. $\mathrm{Ag} / \mathrm{AgCl} / 3 \mathrm{M} \mathrm{KCl}[1.23 \mathrm{~V}$ vs. $\mathrm{RHE}$, reversible hydrogen electrode].

\section{Results and Discussion}

\section{Phase structure}

GIXRD study was performed to know the crystallinity/crystal phase of ZnO NRs (ZNR) sample. The XRD patterns (Figure 1) of the sample confirmed the formation of hexagonal wurtzite

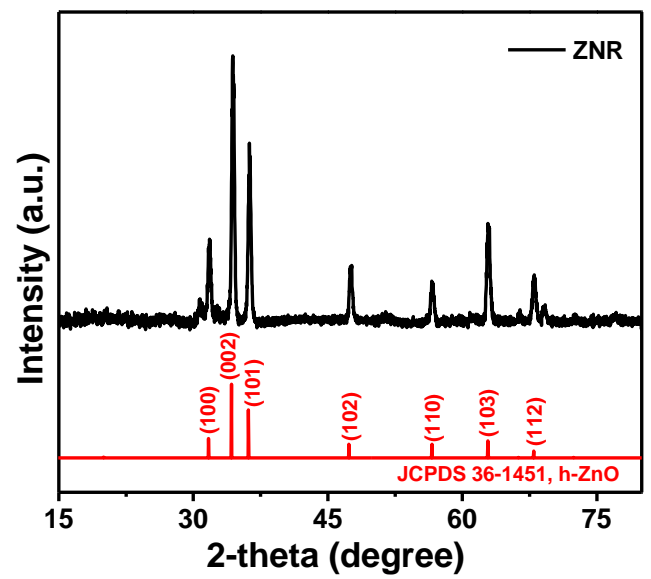

Figure 1 X-ray diffraction pattern of $\mathrm{ZnO}$ nanorods cured at $400^{\circ} \mathrm{C}$. 
$\mathrm{ZnO}(\mathrm{h}-\mathrm{ZnO})$ at $400^{\circ} \mathrm{C}$ curing temperature. The XRD peaks at $2 \theta$ values of $\sim 31.8^{\circ}, \sim 34.4^{\circ}, \sim 36.2^{\circ}, \sim 47.4^{\circ}, \sim 56.5^{\circ}, \sim 62.9^{\circ}$ and $\sim 68^{\circ}$ were assigned (JCPDS Card No. 36-1451) to h-ZnO crystal planes of (100), (002), (101), (102), (110), (103) and (112), respectively. It was found that the intensity of the diffraction peak at $2 \theta$ value of $\sim 34.4^{\circ}$ for the crystal plane (002) was predominant over the other diffraction peaks indicating the preferred growth of $\mathrm{h}-\mathrm{ZnO}$ along c-axis direction. ${ }^{[10]}$

\section{Morphology and microstructure \\ FESEM study}

The FESEM study was carried out to understand the surface morphology of the $\mathrm{ZnO}$ NRs sample cured at $400{ }^{\circ} \mathrm{C}$. The nanorods (NRs) arrays were grown on the $\mathrm{ZnO}$ seed layer coated FTO glass substrate with different refluxing times. It is clearly seen from the FESEM image (Figure 2a) that the $\mathrm{ZnO}$ NRs start to grow at a refluxing time $0.5 \mathrm{~h}$, but the NRs were deposited sparsely with a shorter width and length. However, at $1 \mathrm{~h}$ refluxing time, these were grown vertically onto the seeded layer (Figure $2 \mathrm{~b}$ ) and the rods were further grown considerably at $2 \mathrm{~h}$ refluxing time (Figures $2 \mathrm{c}$ and $2 \mathrm{~d}$ ). Moreover, a thickly populated $\mathrm{ZnO} N R s$ were found with increasing the further refluxing time. The width of the $\mathrm{ZnO}$ NRs was obtained from the top view FESEM images of the ZNR sample grown at $2 \mathrm{~h}$ refluxing time (Figures $2 \mathrm{c}$ and $2 \mathrm{~d}$ ). The width of the rods was $95 \pm 20 \mathrm{~nm}$.
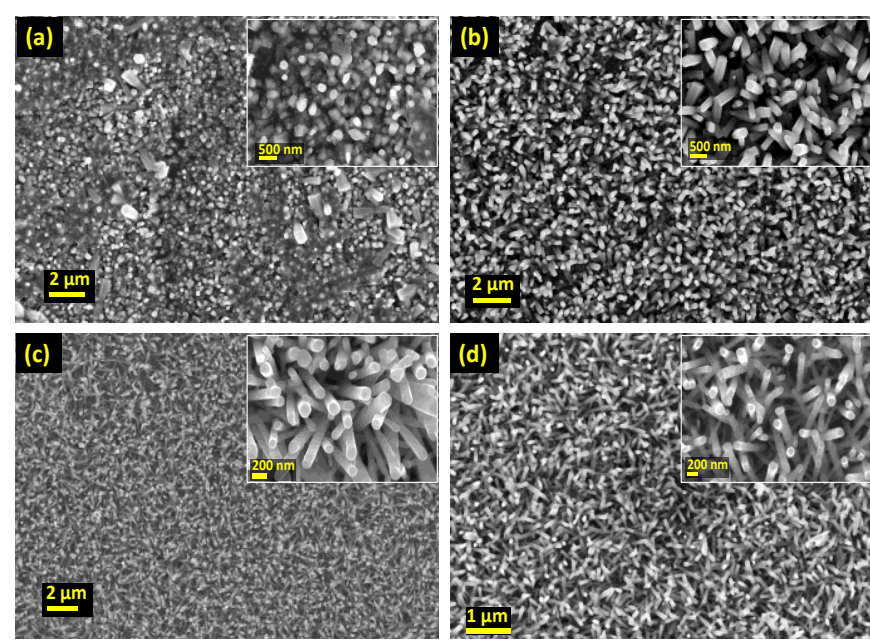

Figure 2 FESEM images of $\mathrm{ZnO}$ nanorods grown on $\mathrm{ZnO}$ seed layer coated FTO glass substrate from aqueous solution of $\mathrm{Zn}\left(\mathrm{NO}_{3}\right)_{2}$ and $\mathrm{HMTA}$ at $95^{\circ} \mathrm{C}$ for different refluxing time: (a) $0.5 \mathrm{~h}$, (b) $1 \mathrm{~h}$ and (c, d) $2 \mathrm{~h}$. Insets (a to d) are the FESEM images of the corresponding samples at higher magnification.

It was worthy to note that the colour of $\mathrm{ZnO}$ NRs sample (ZNR) was white that turned into slightly black after curing the sample under reduce gas atmosphere. This observation was also reported in some published papers. ${ }^{[2,6]}$ The change in colour could be attributed to an increase in oxygen defects in ZNRH sample. ${ }^{[2]}$ Morphological characteristic of ZnO NRs was also studied by FESEM (Figure 3). However, the FESEM image of $\mathrm{ZnO} \mathrm{NRs}$ in $\mathrm{ZNRH}$ sample (Figure $3 \mathrm{~b}$ ) showed a similar morphology to that of ZNR.

\section{TEM study}

Microstructure of the scratched off ZNR sample was analyzed by TEM study (Figure 4). Dimension of the ZnO NRs is distinctly observed from the bright field TEM image (Figure 4a) of the sample. The length and width of the NRs were obtained from the TEM image (Figure 4a). It was found that the length and width of the rods were $\sim 1.5 \mu \mathrm{m}$ and $90 \pm 20 \mathrm{~nm}$, respectively. As displayed in Figures $4 \mathrm{~b}$ and $4 \mathrm{c}$, the HRTEM images of the

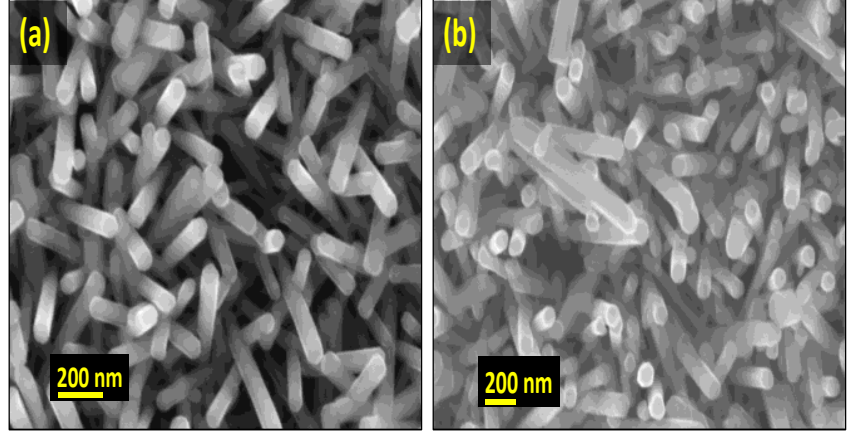

Figure 3 FESEM images of ZnO NRs cured (a) under air atmosphere and (b) reduced gas atmosphere $\left(5 \% \mathrm{H}_{2}\right)$.
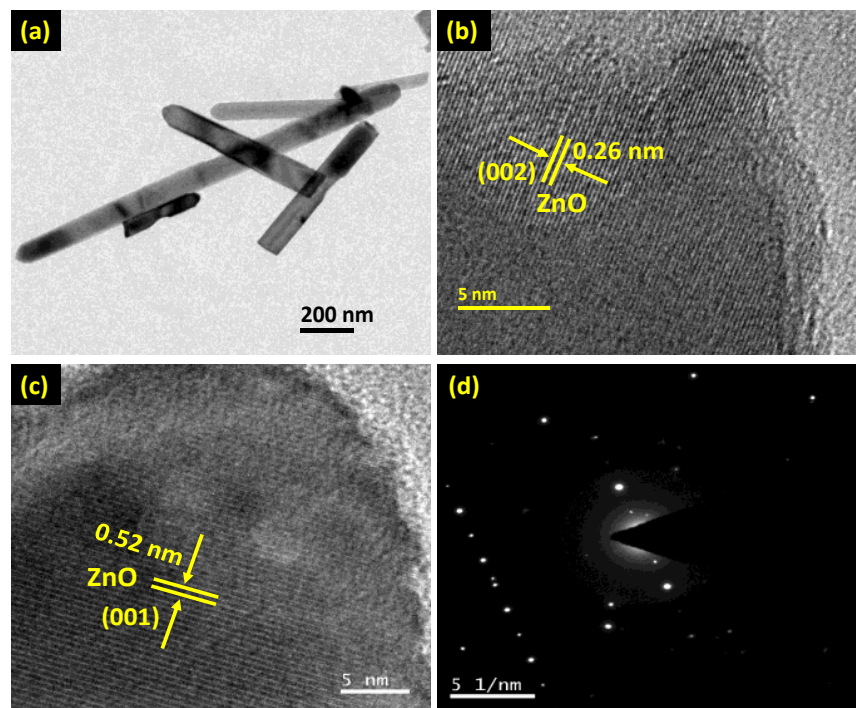

Figure 4 TEM images of scratched off ZNR film prepared at 2 $\mathrm{h}$ refluxing time: (a) Bright field TEM image and (b, c) corresponding HRTEM images of $\mathrm{ZnO}$ nanorods. (d) Displays the SAED pattern of the sample.

ZNR sample show clear lattice fringes with the interplanar d-spacing of $\sim 0.26$ and $\sim 0.52 \mathrm{~nm}$, correspond to $\mathrm{h}$ - $\mathrm{ZnO}$ crystal planes, (002) and (001), respectively. ${ }^{[1,10]}$ The selected-area electron diffraction (SAED) pattern (Figure 4d) also showed the crystalline nature of the sample. Thus, the TEM analysis fully supported the XRD result (Figure 1).

Moreover, the TEM microstructural study of ZNRH sample was further performed to observe the presence of oxygen defects in NRs. It was noteworthy that the structural defects (especially oxygen defects) were clearly visible from the bright field TEM image of ZNRH sample (Figure $5 \mathrm{~b}$ ). It was further noted that the defects were uniformly distributed throughout the entire nanorods. The structural defects had already been noticed by Kegel et al. ${ }^{[4]}$

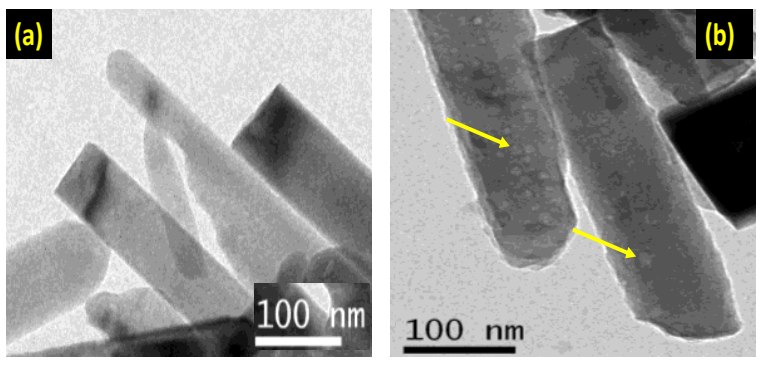

Figure 5 Bright field TEM images of ZnO NRs cured (a) under air atmosphere and (b) reduced gas atmosphere. 


\section{Report}

\section{XPS analysis}

X-ray photoelectron spectral analysis was done to investigate the oxidation states of the constituent elements/oxygen defect (deficiency) in the samples. The XPS binding energy curves for ZNR and ZNRH samples are displayed in Figures $6 a-6 b$ and Figures $6 c-6 d$, respectively. Figure $6 a$ shows strong binding energy peaks centered at $\sim 1021.1$ and $\sim 1044.2$ $\mathrm{eV}$, corresponding to the core-level binding energy signals of $Z n 2 p_{3 / 2}$ and $Z n 2 p_{1 / 2}$, respectively. The energy difference of $\sim 23.1 \mathrm{eV}$ between $Z n 2 p_{1 / 2}$ and $Z n 2 p_{3 / 2}$ core levels, confirmed the presence of $\mathrm{Zn}^{2+}$ ions in ZNR sample. ${ }^{[14]}$ Similar result was noticed in ZNRH sample (Figure 6c). On the other hand, the O1s signal (Figure 6b) of ZNR sample could be split into three Gaussian fitted peaks centered at $\sim 529.8, \sim 530.7$ and $\sim 531.5$ $\mathrm{eV}$, correspondeding to lattice oxygen $\left(\mathrm{O}_{\text {lattice }}\right)$, oxygen deficient region $\left(\mathrm{O}_{\text {defect }}\right)$ and hydroxyl group $\left(\mathrm{O}_{\text {hydroxyl }}\right)$, respectively. ${ }^{[14,15]}$ Three Gaussian fitted peaks of O1s signals (Figure 6d) were also observed in ZNRH sample. The relative contents (\%) of $\mathrm{O}_{\text {lattice }}, \mathrm{O}_{\text {defect }}$ and $\mathrm{O}_{\text {hydroxyl }}$ were calculated from the peak area of O1s signals for both the samples. The calculated relative content (\%) of $\mathrm{O}_{\text {lattice }}, \mathrm{O}_{\text {defect }}$ and $\mathrm{O}_{\text {hydroxyl }}$ for ZNR sample were $\sim 73 \%, \sim 4 \%$ and $\sim 23 \%$, respectively. The same for ZNRH sample were $\sim 43 \%, \sim 36 \%$ and $\sim 21 \%$, respectively. Thus, the content (\%) of oxygen defect/deficiency in the ZNRH sample was $\sim 9$ times higher than the ZNR sample. This observation could be attributed to the removal of lattice oxygen in $\mathrm{h}-\mathrm{ZnO}$ lattice by post annealing of ZNR sample under the reducing atmosphere $\left(5 \% \mathrm{H}_{2}\right)$ at $350{ }^{\circ} \mathrm{C} \cdot{ }^{[6]}$ The higher content $(\%)$ of oxygen deficiency would facilitate to absorb more visible light. This could be beneficial for a material towards an enhancement of PEC property. ${ }^{[14]}$
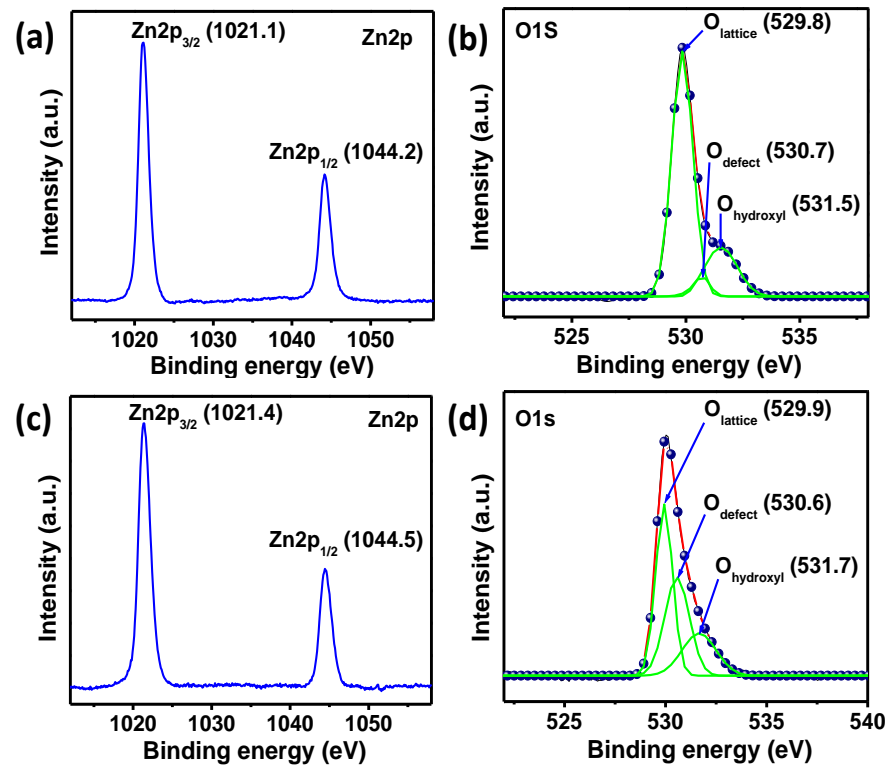

Figure 6 XPS binding energy curves: (a) Zn2p, (b) O1s of ZNR; (c) Zn2p, (d) O1s of ZNRH film samples.

\section{Photoluminescence (PL) spectral study}

The PL spectral study (Figure 7) of ZNR and ZNRH samples was also performed. As seen in Figure 7, the PL spectrum of ZNR shows a strong emission peak centered at $\sim 385 \mathrm{~nm}$ ascribed to the band edge emission (BEE) of hexagonal wurtzite $\mathrm{ZnO}$ semiconductor ${ }^{[14]}$ Moreover, in this sample, two other prominent emission peaks observed at $\sim 424$ and $\sim 486 \mathrm{~nm}$, attributed to zinc interstitials and antisite oxygen, respectively. ${ }^{[11]}$ It was worthy to note that in ZNRH sample, a very strong

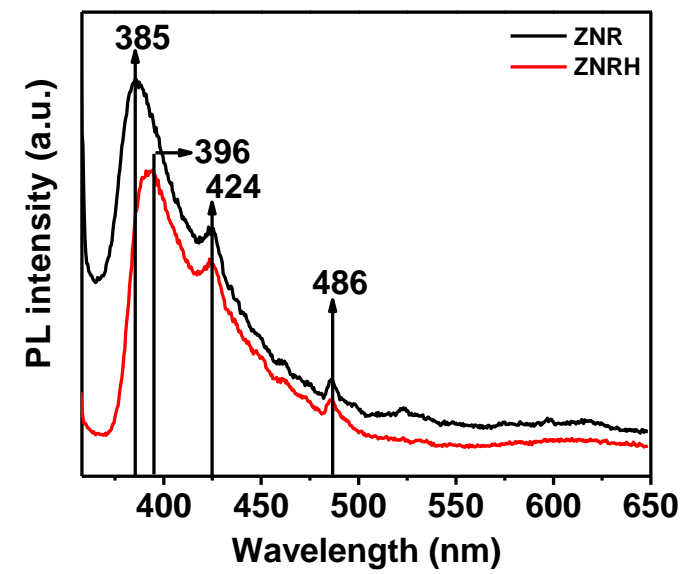

Figure 7 Photoluminescence (PL) emission spectra $\left(\lambda_{\text {ex }}=340\right.$ $\mathrm{nm}$ ) of ZNR and ZNRH films.

intensity emission peak was noticed at $\sim 396 \mathrm{~nm}$. In this sample, the BEE peak for the semiconductor with reduced intensity was seemed to be disguised within the strong intensity emission peak appeared at $396 \mathrm{~nm}$. As found in ZNR, two other prominent emissions at $\sim 424$ and $\sim 486 \mathrm{~nm}$ were also observed in ZNRH film. In our previous report, ${ }^{[11]}$ we found a strong intensity emission peak at $\sim 398 \mathrm{~nm}$ in nanostructured Al doped $\mathrm{ZnO}$ thin film on quartz glass. In the reported work, with the help of PL emission and Raman spectra, XPS studies and measurement of magnetic property, we established that the emission peak was due to paramagnetic singly negatively charged oxygen vacancy. ${ }^{[11]}$ It was also seen that this special type of oxygen defect could play an effective role in the photocatalytic decomposition of organic dye like Rhodamine 6G even under visible light exposure. In the present work, it is believed that the emission peak found at $\sim 396 \mathrm{~nm}$ could be due to the existence of singly negatively charged oxygen vacancy, generated in the $\mathrm{ZnO}$ matrix due to the effect of reduced gas atmosphere. ${ }^{[2,6]}$ This particular paramagnetic defect with zinc interstitials and antisite oxygen defects as observed particularly in ZNRH could enhance the PEC activity of $\mathrm{ZnO}$ nanorods (discussed later).

\section{Light harvesting efficiency (LHE)}

The LHE for ZNR and ZNRH samples were measured by employing integrating sphere, a special attachment of the UV-Vis spectrophotometer. As seen in Figure 8, an enhanced $\mathrm{LHE}$ was found in the visible wavelength region of ZNRH sample. The improved LHE value of ZNRH sample compared to ZNR sample could be associated with an enhanced oxygen

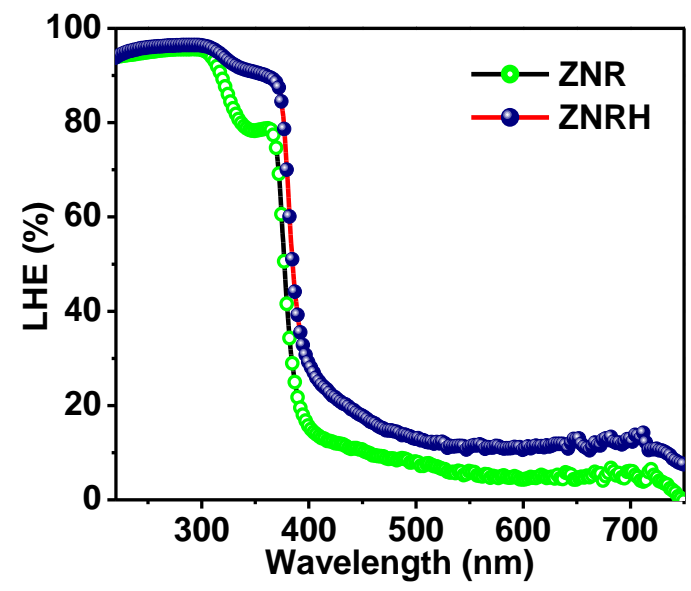

Figure 8 Light harvesting efficiency of the samples. 
deficiencies especially the presence of singly negatively charged oxygen vacancy (as ascertained already by PL spectra, Figure 7; XPS study, Figure 6), which could be generated by the treatment of the sample under reducing hydrogen gas atmosphere. ${ }^{[6]}$ It was worthy to note that the LHE value was calculated from the reflection $(R)$ and transmission $(T)$ value of the samples by using Eq. (1). ${ }^{[16]}$ Thus, it was possible to make an efficient photoanode with improved PEC activity by using this facile strategy.

$$
\operatorname{LHE}(\lambda)(\%)=100 \%-\mathrm{R}(\lambda)(\%)-\mathrm{T}(\lambda)(\%)
$$

\section{Photoelectrochemical (PEC) activity}

The PEC performance of ZNR and ZNRH samples was investigated by using a PEC cell with three electrode systems under visible light irradiation. In this study, aqueous solution of $0.1 \mathrm{M} \mathrm{Na}_{2} \mathrm{SO}_{4}$ was used as an electrolyte. The linear sweep voltagram curves (LSV, Figures $9 \mathrm{a}$ and $9 \mathrm{~b}$ ) of the samples were recorded from the voltage range of $-0.4 \mathrm{~V}$ to $1.0 \mathrm{~V} v \mathrm{vs}$. $\mathrm{Ag} / \mathrm{AgCl} / 3 \mathrm{M} \mathrm{KCl}$ reference electrode under visible light exposure (wavelengths, $\geq 400 \mathrm{~nm}$ ). For this purpose, a $300 \mathrm{~W}$ xenon lamp and a PEC cell equipped with water filter of $1 \mathrm{M} \mathrm{NaNO}_{2}$ (aq.) solution were used. ${ }^{[1]}$ In Figure 9a, the LSV curve of ZNRH sample shows a significant increase in anodic photocurrent density (PD) compared to the ZNR sample. Moreover, the LSV curve of ZNRH sample exhibited $\sim 2.0$ times higher PD than ZNR sample. This observation could be attributed to an enhanced light absorption due to higher content (\%) of oxygen vacancy especially singly negatively charged oxygen vacancy in ZNRH that was realized from the PL spectrum (Figure 7) and XPS study (Figure 6) of the samples. ${ }^{[11,14]}$ The LSV curves of both ZNR and ZNRH samples were recorded under light off and on conditions (Figure 9b). Chronoamperometric study (photocurrent vs. time curve, Figure 9c) was performed to check the photostability of the samples. Moreover, the electrochemical impedance spectra (EIS) were also measured to confirm the efficient charge separation and transportation that occurred in the samples. The EIS Nyquist plots (Figure 9d) showed a smaller arc radius for ZNRH sample compared to ZNR sample.
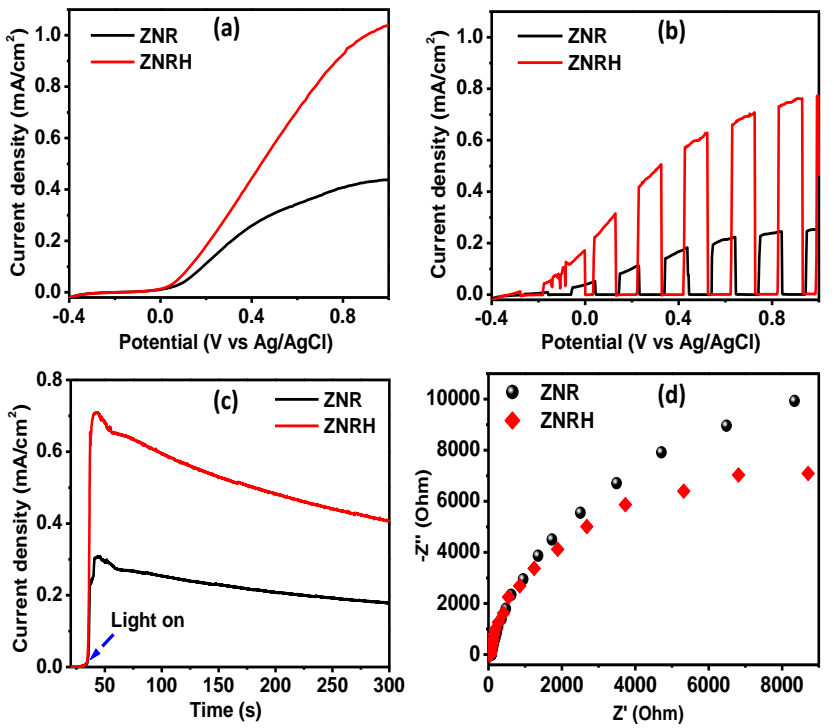

Figure 9 PEC activities of the samples: $(a, b)$ linear sweep voltammetry (LSV) curves measured under constant and chopped irradiation of visible light, respectively; (c) chronoamperometric study (photocurrent density vs. time, l-t curve) of the films recorded under visible light exposure; (d) electrochemical impedance spectra (EIS) (Nynquist plots) of the samples measured under visible light exposure.
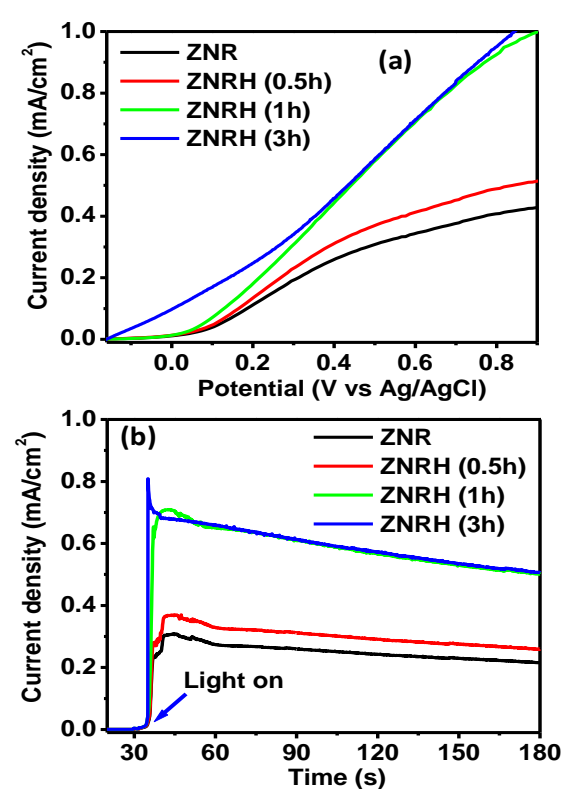

Figure 10 PEC activity of ZnO NRs cured under reduce gas atmosphere at different incubation time under visible light exposure: (a) linear sweep voltammetry (LSV) curves; (b) chronoamperometry study.

This result also confirmed the generation of lower interface layer resistance in ZNRH sample. Thus, an efficient photogenerated charge separation and transportation occurred in $\mathrm{ZNRH}$ sample resulting an increase in its PEC activity. ${ }^{[13,15]}$ It was noted that the influences of incubation time of reduce gas treatment on PEC activity of the sample was also studied up to $3 \mathrm{~h}$. It was found that the PEC activity increased gradually with the incubation time up to $1 \mathrm{~h}$ (Figure 10). The ZNRH sample cured under the reduce gas atmosphere for $1 \mathrm{~h}$ incubation time exhibited the highest PD. This observation could be attributed to the further enhancement of donor density due to increased oxygen defects in the sample. ${ }^{[2-6]}$

\section{Conclusion}

In brief, $\mathrm{ZnO} \mathrm{NRs}$ were grown onto $\mathrm{ZnO}$ seed layer via hydrothermal technique at low temperature $\left(\sim 95^{\circ} \mathrm{C}\right)$ and further heat-treated at $400{ }^{\circ} \mathrm{C}$. To enhance oxygen defects such as oxygen vacancy in the NRs, the thermally cured samples were again heat-treated in hydrogen gas atmosphere. Morphological, microstructural analyses, estimation of oxygen vacancy in the samples were carried out by field emission scanning, transmission and X-ray photo electron microscopies, respectively. Moreover, the optical properties of the specimen were also characterized by photoluminescence measurement. A co-relation was also made between the reduced gas treatment time and the PEC activity of the samples. The ZnO NRs synthesized by optimizing the time for the growth of NRs as well as the reduced gas treatment time exhibited significant improvement in the PEC activity of the sample. This work could make an avenue for enhancing the PEC activity of other hierarchically structured metal oxide semiconductors.

\section{Acknowledgement}

One of the authors, H. K. thankfully acknowledges CSIR, Govt. of India for providing his Ph.D. research fellowship. The authors also acknowledge the help rendered by Electron Microscopy Section for XRD, FESEM and TEM characterizations. The work had been done as an associated research work of $12^{\text {th }}$ Five Year Plan project of CSIR (No. ESC0202). 


\section{Report}

\section{Author Contributions}

The article was written with the help of all authors. All authors have given approval to the final version of the manuscript.

\section{References}

[1] Yu, Y.-X.; Ouyang, W.-X.; Liao, Z.-T.; Du, B.-B.; Zhang W.-D. ACS Appl. Mater. Interfaces 2014, 6, 8467.

[2] Singh, A. P.; Kodan, N.; Mehta, B. R.; Held, A.; Mayrhofer, L.; Moseler, M. ACS Catal. 2016, 6, 5311.

[3] Hou, Y.; Zuo, F.; Dagg, A.; Feng, P. Nano Lett. 2012, 12, 6464.

[4] Kegel, J.; Zubialevich, V. Z.; Schmidt, M.; Povey, I. M.; Pemble, M. E. ACS Appl. Mater. Interfaces 2018, 10, 17994.

[5] Kegel, J.; Laffir, F.; Povey, I. M.; Pembleac, M. E. Phys. Chem. Chem. Phys. 2017, 19, 12255.

[6] Cooper, J. K.; Ling, Y.; Longo, C.; Li, Y.; Zhang, J. Z. J. Phys. Chem. C 2012, 116, 17360.

[7] Zhang, S.; Wei, S. H.; Zunger, A. Phys. Rev. B 2001, 63, 075205.

[8] Lany, S.; Zunger, A. Phys. Rev. B 2005, 72, 035215.
[9] Wang, G; Wang, H.; Ling, Y.; Tang, Y.; Yang, X.; Fitzmorris, R. C.; Wang, C.; Zhang, J. Z.; Li, Y. Nano Lett. 2011, 11, 3026.

[10] Yang, X.; Li, H.; Zhang, W.; Sun, M.; Li, L.; Xu, N.; Wu, J.; Sun, J. ACS Appl. Mater. Interfaces 2017, 9, 658.

[11] Pal, M.; Bera, S.; Sarkar, S.; Jana, S. RSC Adv. 2014, 4, 11552.

[12] Han, C.; Chen, Z.; Zhang, N.; Colmenares, J. C.; Xu, Y.-J. Adv. Funct. Mater. 2015, 25, 221.

[13] Bera, S.; Pal, M.; Sarkar, S.; Jana, S. ACS Appl. Mater. Interfaces 2017, 9, 4420.

[14] Bera, S.; Khan, H.; Biswas, I.; Jana, S. Appl. Surf. Sci. 2016, 383, 165.

[15] Khan, H.; Bera, S. Sarkar, S. Jana, S. Surf. Coat. Technol. 2017, $328,410$.

[16] Khan, H.; Seth, M.; Naskar, A.; Jana, S. J. Sol-Gel Sci. Technol. 2018, 88, 359.

Received August 19, 2019 Accepted October 9, 2019 